Achim Hamann

Energieeffiziente Nichtwohngebäude

Grundlagen, Beispiele und Bilanzierungsansätze nach DIN V 18599
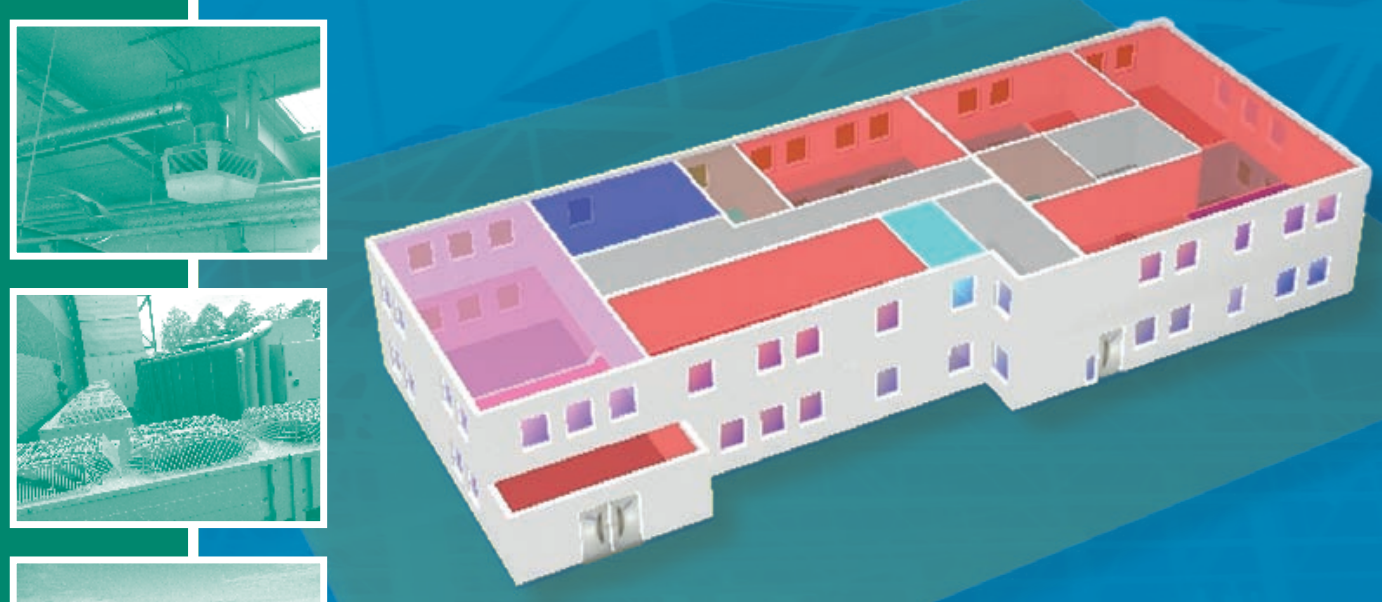

Fraunhofer IRB Verlag

(1)

Generiert durch IP '172.22.53.54', am 26.04.2023, 10:24:12 
Achim Hamann

Energieeffiziente Nichtwohngebäude 
Achim Hamann

\section{Energieeffiziente Nichtwohngebäude}

Grundlagen, Beispiele und Bilanzierungsansätze nach DIN V 18599

Fraunhofer IRB Verlag 
Bibliografische Information der Deutschen Nationalbibliothek:

Die Deutsche Nationalbibliothek verzeichnet diese Publikation in der

Deutschen Nationalbibliografie; detaillierte bibliografische Daten sind

im Internet über www.dnb.de abrufbar.

ISBN (Print): 978-3-8167-9768-5

ISBN (E-Book): 978-3-8167-9840-8

Umschlaggestaltung: Martin Kjer

Herstellung: Andreas Preising

Satz: Fotosatz Buck, Kumhausen/Hachelstuhl

Druck: Bosch-Druck GmbH, Ergolding

Alle Rechte vorbehalten.

Dieses Werk ist einschließlich aller seiner Teile urheberrechtlich geschützt. Jede Verwertung, die über die engen Grenzen des Urheberrechtsgesetzes hinausgeht, ist ohne schriftliche Zustimmung des Fraunhofer IRB Verlages unzulässig und strafbar. Dies gilt insbesondere für Vervielfältigungen, Übersetzungen, Mikroverfilmungen sowie die Speicherung in elektronischen Systemen.

Die Wiedergabe von Warenbezeichnungen und Handelsnamen in diesem Buch berechtigt nicht zu der Annahme, dass solche Bezeichnungen im Sinne der Warenzeichen- und MarkenschutzGesetzgebung als frei zu betrachten wären und deshalb von jedermann benutzt werden dürften. Sollte in diesem Werk direkt oder indirekt auf Gesetze, Vorschriften oder Richtlinien (z. B. DIN, VDI, VDE) Bezug genommen oder aus ihnen zitiert werden, kann der Verlag keine Gewähr für Richtigkeit, Vollständigkeit oder Aktualität übernehmen. Es empfiehlt sich, gegebenenfalls für die eigenen Arbeiten die vollständigen Vorschriften oder Richtlinien in der jeweils gültigen Fassung hinzuzuziehen.

(C) Fraunhofer IRB Verlag, 2017

Fraunhofer-Informationszentrum Raum und Bau IRB

Nobelstraße 12, 70569 Stuttgart

Telefon +49 $711970-2500$

Telefax +49711 970-2508

irb@irb.fraunhofer.de

www.baufachinformation.de 


\section{Inhaltsverzeichnis}

Vorwort. ........................ 11

\section{Teil I: Einführung}

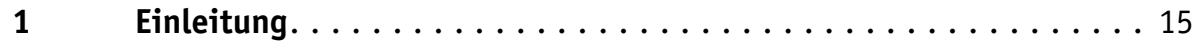

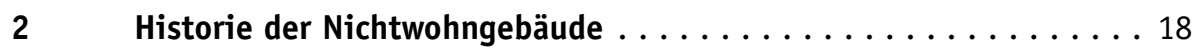

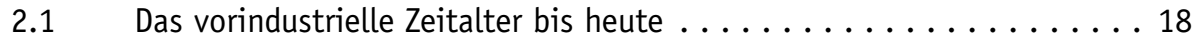

2.2 Das Industriezeitalter und die Moderne . . . . . . . . . . 21

2.3 Die heutige Dienstleistungsgesellschaft .............. 25

2.4 Großflächige Nichtwohngebäude des Handels und Gewerbes. . . . . . . . 27

2.5 Heutige Sanierungsprojekte ................... 28

2.6 Zusammenfassung zum Sammelsurium der Gebäudetypen. . . . . . . . . 29

3 Von den Anfängen des Wärmeschutzes über KfW bis BIM . . . . . 33

3.1 1929: Die DIN V $4701 \ldots \ldots \ldots \ldots \ldots \ldots \ldots \ldots \ldots \ldots$

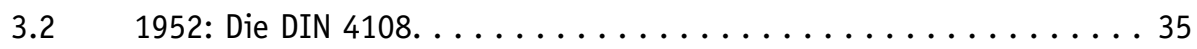

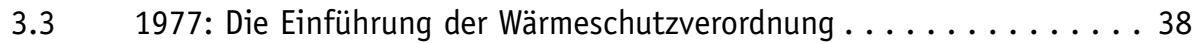

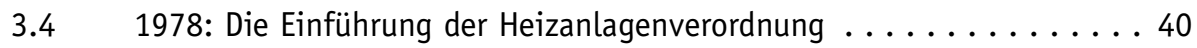

3.5 2002: Die Geburtsstunde der Energieeinsparverordnung. . . . . . . . 42

3.6 2005: Die Vornorm DIN V 18599 erblickt das Licht der Welt. . . . . . . 47

3.7 2009: Das Erneuerbare Energien Wärmegesetz, ein Meilenstein. . . . . 50

3.8 2015: Die KfW-Effizienzhausförderung für Nichtwohngebäude ..... 53

3.9 2016: Aktuelle Randbedingungen der Bilanzierung. . . . . . . . . 55

3.10 Ab 2017: Building Information Modeling und energieeffizientes Bauen . 56

$4 \quad$ Politische Zielsetzungen, Handlungsempfehlungen für die Stadtebene........................60 60

4.1 2019: Niedrigstenergiegebäude und klimaneutraler Gebäudebestand . . 60

4.2 1990-2050: $\mathrm{CO}_{2}$-Reduzierung um 80 bis $90 \%$ und Szenarien . . . . . 61

4.3 2010-2050: Handlungsempfehlungen für die Stadtebene. . . . . . . . 67

4.4 2016: Das »Klima-Profit-Netzwerk« als Instrument zur Modernisierung des Nichtwohngebäudebestands . . . . . . . . . . . 75

4.5 Nach 2016: Übergang auf die Gebäudeebene bzw. zum Effizienzhausstandard ....................... 76

4.6 Zusammenfassung und Überleitung zu Teil II. . . . . . . . . 77

Teil II: Leitfaden

5 Die wichtigsten Planungsgrundlagen und ihre Einflüsse auf die

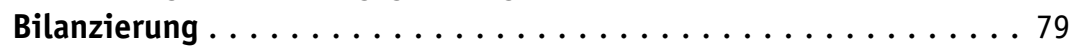

5.1 Exkurs: Modellgebäude zu Nichtwohngebäudetypen ......... 81 
$5.2 \quad$ Planungsgrundlage EnEV . . . . . . . . . . . . . 84

5.2.1 Anrechnung von Strom und Einfluss auf die Bilanzierung . . . . . . 84

5.2.2 Die Luftdichtheit und ihr Einfluss auf die Bilanzierung . . . . . . 86

5.2.3 Mindestwärmeschutz und Einfluss von Wärmebrücken . . . . . . . . 87

5.2.4 Vergleich: Energetische Verbesserung der Gebäudehülle um $20 \% \ldots 89$

5.2.5 Einfluss des Warmwasserbedarfs auf die Bilanzierung ........ 90

5.2.6 Die Nettogrundfläche und der Einfluss auf die Bilanzierung . . . . . 91

5.2.7 Das Referenzgebäude, eine zu überwindende Hürde ......... 92

5.2.8 Appell zur konsequenten Fortschreibung der Unterlagen im Bauprozess. 93

5.2.9 Die Nutzungsprofile der DIN V 18599 und individuelle Ansätze. . . . . . 93

5.2.10 Der Umgang mit Erdberührung und Bauteilen gegen unbeheizte Räume. 94

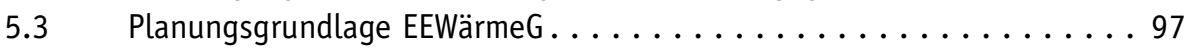

5.4 Planungsgrundlage DIN 4108: Wärmeschutz und Energie-Einsparung in Gebäuden............................. 103

5.4.1 DIN 4108 Teil 2: Mindestanforderungen an den Wärmeschutz, an Wärmebrücken, an den sommerlichen Wärmeschutz und Anforderungen an die Luftdichtheit sowie Rollladenkästen. . . . . . . . . 103

5.4.2 DIN 4108 Teil 3: Klimabedingter Feuchteschutz - Anforderungen, Berechnungsverfahren und Hinweis für Planung und Ausführung . . . 109

5.4.3 DIN 4108 Teil 4: Wärme- und Feuchteschutz, Bemessungswerte . . . . 111

5.4.4 DIN V 4108 Teil 6: Sommerlicher Wärmeschutz und Wärmeverlust über das Erdreich . . . . . . . . . . . . . . . . . . . 112

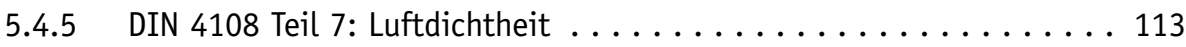

5.4.6 DIN 4108 - Beiblatt 2: Wärmebrücken ................ 115

5.5 Anmerkungen zur DIN 4701-10: Energetische Bewertung heiz- und raumlufttechnischer Anlagen . . . . . . . . . . . . . . . . 117

5.6 Planungsgrundlage DIN EN ISO 6946: Bauteile - Wärmedurchlasswiderstand und Wärmedurchgangskoeffizient - Berechnungsverfahren . . 117

5.7 Grundlagen und Einflüsse auf die Bilanzierung bei Anwendung der DIN EN ISO 13370: Wärmetechnisches Verhalten von Gebäuden Wärmeübertragung über das Erdreich . . . . . . . . . . . 119

5.8 Anmerkungen zur DIN EN ISO 13788: Wärme- und feuchtetechnisches Verhalten von Bauteilen und Bauelementen . . . . . . . . 127

5.9 Grundlagen nach DIN EN ISO 13789: Wärmetechnisches Verhalten von Gebäuden - Spezifischer Transmissions- und Lüftungswärmedurchgangskoeffizient - Berechnungsverfahren . . . . . . . . 128

5.10 Grundlagen nach DIN EN ISO 13790: Energieeffizienz von Gebäuden - Berechnung des Energiebedarfs für Heizung und Kühlung . . . . . . . 131

5.11 Grundlagen nach DIN EN ISO 10211: Wärmebrücken im Hochbau Wärmeströme und Oberflächentemperaturen ............ 133 
5.12 Einflüsse auf die Bilanz und Erläuterungen zur Bilanzierung nach DIN V 18599 Teil 1 bis 11: Energetische Bewertung von Gebäuden . . . 142

5.12.1 KfW-Anforderungen an ein Effizienzhaus und die Bilanzierung . . . . . 143

5.12.2 Grundlagen nach DIN V 18599-1: Allgemeine Bilanzierungsverfahren, Begriffe, Zonierung, Bewertung der Energieträger . . 145

5.12.3 Grundlagen nach DIN V 18599-2: Nutzenergiebedarf für Heizen und Kühlen von Gebäudezonen . . . . . . . . . . . . . . . . . . . . . 146

5.12.4 Einfluss von Varianten zu Lüftungsanlagen nach DIN V 18599-3: Nutzenergiebedarf für die energetische Luftaufbereitung. . . . . . . . . . 149

5.12.5 Einfluss von Varianten zur Beleuchtung nach DIN V 18599-4: Nutzund Endenergiebedarf für Beleuchtung . . . . . . . . . . . . . . . . . 154

5.12.6 Einfluss von Varianten zu Heizsystemen und Wärmepumpen nach DIN V 18599-5: Endenergiebedarf von Heizsystemen . . . . . . . . . 158

5.12.7 Anmerkungen zur DIN V 18599-6: Endenergiebedarf von Lüftungsanlagen, Luftheizungsanlagen und Kühlsystemen für den Wohnungsbau 170

5.12.8 Einfluss von Lüftungsanlagen in Kombination mit Heizen und Kühlen nach DIN V 18599-7: Endenergiebedarf von Raumlufttechnik- und Klimakältesystemen für den Nichtwohnungsbau. . . . . . 170

5.12.9 Einfluss der Warmwasserbereitung nach DIN V 18599-8: Nutz- und Endenergiebedarf von Warmwasserbereitungssystemen . . . . . . . . . 184

5.12.10 Einfluss von Blockheizkraftwerken nach DIN V 18599-9: End- und Primärenergiebedarf von stromproduzierenden Anlagen . . . . . . . . . 188

5.12.11 Erläuterungen zur DIN V 18599-10: Nutzungsrandbedingungen, Klimadaten . . . . . . . . . . . . . . . . . . . . 190

5.12.12 Erläuterungen zur DIN V 18599-11: Gebäudeautomation . . . . . . . . . 191

5.12.13 Anmerkungen zu DIN V 18599 Beiblatt 1: Bedarfs-/Verbrauchsabgleich 194

5.12.14 Anmerkungen zu DIN V 18599 Beiblatt 2: Beschreibung zu Kennwerten bei Nachweisen zum EEWärmeG . . . . . . . . . . . . . . 194

5.13 Zusammenfassung und Überleitung zu Beispielen. . . . . . . . . . . . . 195

6 Umsetzen des KfW-Effizienzhaus-Standards an Beispielen . . . . . 196

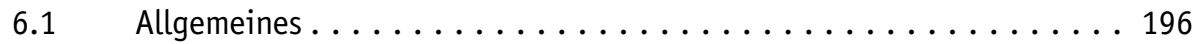

6.2 Das Bürogebäude . . . . . . . . . . . . . . . . . . . 197

6.2.1 Allgemeine Gebäudebeschreibung . . . . . . . . . . . . . . . . . 197

6.2.2 Beschreibung der Gebäudehülle des Bürogebäudes . . . . . . . . . . . . . . 198

6.2.3 Beschreibung der Gebäudetechnik des Bürogebäudes . . . . . . . . . . 202

6.2 .4 Ergebnisse............................. 203

6.3 Das Hotel mit Gastronomie. . . . . . . . . . . . . . . . . . 205

6.3.1 Allgemeine Gebäudebeschreibung und Einteilung in Zonen . . . . . . . 205

6.3.2 Beschreibung der Gebäudehülle des Hotels . . . . . . . . . . . . . . . 205

6.3.3 Beschreibung der Gebäudetechnik des Hotels . . . . . . . . . . . 206 


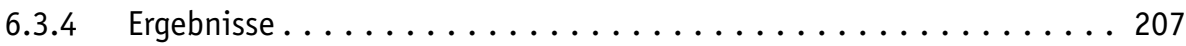

6.4 Das Produktionsgebäude mit Verwaltung............. 209

6.4.1 Allgemeine Gebäudebeschreibung und Einteilung in Zonen ....... 209

6.4.2 Beschreibung der Gebäudehülle des Produktionsgebäudes . . . . . . 210

6.4.3 Beschreibung der Gebäudetechnik des Produktionsgebäudes . . . . . 212

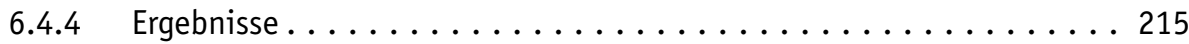

6.5 Großflächige Handelsgebäude: $17^{\circ} \mathrm{C}$ Innenraumtemperatur, keine Kühlprodukte . . . . . . . . . . . . . . . . . . . 217

6.5.1 Allgemeine Gebäudebeschreibung und Einteilung in Zonen . . . . . 217

6.5.2 Beschreibung der Gebäudehülle.................. 218

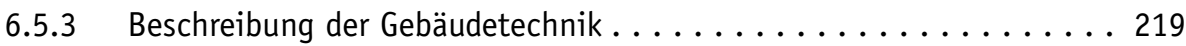

6.5 .4 Ergebnisse......................... 220

6.6 Großflächige Handelsgebäude: $21^{\circ} \mathrm{C}$ Innenraumtemperatur, mit Kühlprodukte ........................ 221

6.6.1 Allgemeine Gebäudebeschreibung und Einteilung in Zonen ...... 221

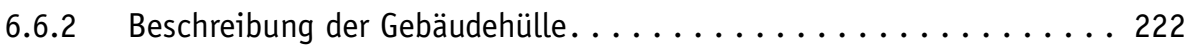

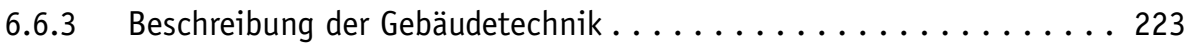

6.6 .4 Ergebnisse............................ 224

6.7 Die Logistik- und Gewerbehalle mit Verwaltung ............ 226

6.7.1 Allgemeine Gebäudebeschreibung und Einteilung in Zonen . . . . . 226

6.7.2 Beschreibung der Gebäudehülle. . . . . . . . . . . . . . . 227

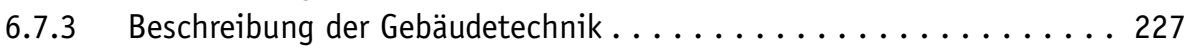

6.7 .4 Ergebnisse......................... 230

6.8 Zusammenfassung und Überleitung zu Teil III . . . . . . . . . 231

Teil III: Immobilienökonomie und sonstige Trends mit Blick auf einen nachhaltigen Nichtwohngebäudebestand

7 Modellansatz zur Abschätzung des Investitionsvolumens auf der städtischen Ebene zur energetischen Modernisierung des Nichtwohngebäudebestands .................... 233

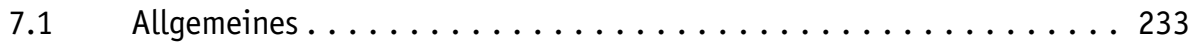

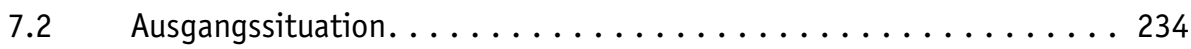

$7.3 \quad$ Stand der Forschung . . . . . . . . . . . . . . 235

7.4 Vorgehensweise und Zielsetzung am Beispiel Wuppertal-Vohwinkel... 236

7.5 Methodische Vorgehensweise beim Modellieren des Bestands . . . . . 238

7.6 Beschreibung des Modellansatzes für die Stadtteilebene . . . . . . 245

7.7 Ergebnisse bei Anwendung des Modells auf einen Stadtteil und die Hochrechnung auf Stadtebene am Beispiel Wuppertal. . . . . . . . . 247

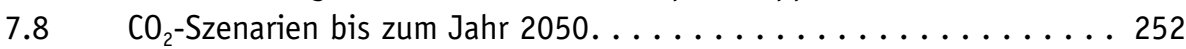

7.9 Nutzen für immobilienökonomische Betrachtungen. . . . . . . 255 
7.10 Notwendiges Investitionsvolumen am Beispiel von Wuppertal-

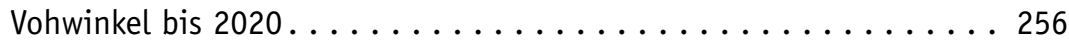

8 Trends und zukunftsfähige Lösungsansätze . . . . . . . . . . . . 267

8.1 Virtuelle Kraftwerke und Energiespeicher in Gebäuden . . . . . . . . . 268

8.2 Weitere geothermische Möglichkeiten. . . . . . . . . . . . 270

8.3 Power to Gas . . . . . . . . . . . . . . . . . . . . 272

8.4 Bauteile aus PCM - Phase Change Materials . . . . . . . . . . . . 272

8.5 Sonstige Bilanzierungsansätze zu nachhaltigen Gebäuden . . . . . . . . 273

$8.6 \quad$ Smart City . . . . . . . . . . . . . . . . . . . . . . 274

Teil IV: Ausblick

Abbildungsverzeichnis . . . . . . . . . . . . . . . . . 279

Tabellenverzeichnis . . . . . . . . . . . . . . . . . . . 282

Abkürzungsverzeichnis. . . . . . . . . . . . . . . . . 283

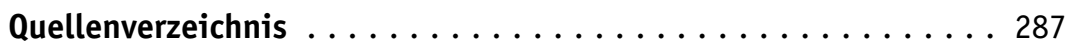

Liste der Normen bzw. Grundlagen im Teil »Leitfaden« . . . . . . . 292

Stichwortverzeichnis . . . . . . . . . . . . . . . . 295 
